\title{
Teorías subjetivas del comportamiento prosocial: significados, desarrollo y motivaciones de jóvenes voluntarios ante un desastre socionatural
}

\section{Subjective theories of prosocial behavior: Meanings, development and motivations of young volunteers in the face of a socionatural disaster}

\author{
Robert Alvarado Ardiles ${ }^{a}$, Constanza Pradenas Ossandón ${ }^{a}$, Nátaly Yañez Vega ${ }^{a}$, \\ David Cuadra Martínez ${ }^{\mathrm{b}}$ José Sandoval Díaz, \\ aUniversidad Santo Tomás, Chile \\ bUniversidad de Atacama, Chile \\ 'Universidad del Bío Bío, Chile
}

\section{Resumen}

Objetivo: el presente trabajo busca conocer las teorías subjetivas de la conducta prosocial de jóvenes voluntarios durante las etapas de respuesta y rehabilitación de un desastre socionatural. Método: se utilizó un diseño de caso cualitativo seleccionando, vía muestreo intencionado, 11 estudiantes universitarios voluntarios ante el desastre hidrometeorológico en Atacama, Chile. Los datos son producidos mediante entrevistas semiestructuradas y un grupo de discusión, utilizando como estrategia de análisis el proceso de codificación de la teoría fundamentada. Resultados: encontramos que (i) los voluntarios significan la conducta prosocial como un proceso de ayuda humanitaria, de temporalidad acotada, la cual depende de la empatía hacia los afectados, percepción de (in)capacidad institucional, evaluación subjetiva del riesgo, capacidades autopercibidas y sentido de responsabilidad social ante el desastre; (ii) Su desarrollo se vincula a una formación valórica temprana, la cual es mantenida por la participación constante en el tiempo en este tipo de actividades y el soporte emocional de terceros significativos; y (iii) Las motivaciones para el despliegue de estas conductas de ayuda, son del tipo extrínseco-social e intrínsecos-personales. Conclusiones: se sostiene la importancia del proceso formativo-educativo para el fortalecimiento del comportamiento prosocial, así como también, la importancia de formalizar el proceso de voluntariado ante estos eventos.

Palabras clave: comportamiento prosocial, teorías subjetivas, jóvenes voluntarios, desastre socionatural, motivaciones, significados.

\section{Para citar este artículo:}

Alvarado, R., Pradenas, C., Yañez, N., Cuadra, D., \& Sandoval, J. (2019). Teorías subjetivas del comportamiento prosocial: significados, desarrollo y motivaciones de jóvenes voluntarios ante un desastre socionatural. Liberabit, 25(2), 251-266. https://doi.org/10.24265/liberabit.2019.v25n2.08

\begin{abstract}
Objective: The present work aims to learn about the subjective theories of the prosocial behavior of young volunteers during the response and recovery stages of a socionatural disaster. Method: A qualitative case design was used by selecting, through intentional sampling, 11 university students who volunteered in the face of the hydrometeorological disaster occurred in Atacama, Chile. Data was collected through semi-structured interviews and a discussion group, using the coding process in grounded theory as an analysis strategy. Results: We found that (i) volunteers understand prosocial behavior as a process of limited-temporality humanitarian aid which depends on empathy towards those affected; the perception of institutional (in)capacity; a subjective risk assessment; self-perceived abilities; and a sense of social responsibility in the face of a disaster; (ii) its development is linked to an early value training, which is maintained by the constant participation in this type of activities over time and the emotional support of significant third parties; and (iii) the types of motivation to show this helping behavior are extrinsic-social and intrinsic-personal. Conclusions: The importance of the training-educational process for strengthening prosocial behavior is supported, as well as the importance of formalizing the volunteer recruitment process in the face of these events.
\end{abstract}

Keywords: prosocial behavior, subjective theories, young volunteers, socionatural disaster, motivations, meanings.

Este es un artículo Open Access bajo la licencia Creative Commons Atribución-NoComercial-CompartirIgual 4.0

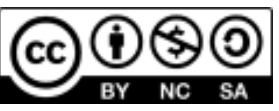




\section{Introducción}

La investigación sobre desastres socionaturales ha evolucionado desde su énfasis inicial en los peligros naturales hacia la construcción social del proceso de riesgo de desastre (García, 2005; Oficina de las Naciones Unidas para Reducción de Riesgo de Desastres [UNISDR], 2015; Wisner, 2016). En la actualidad, los desastres socionaturales, eventos destructivos producidos por la interacción de un peligro natural y la vulnerabilidad espacializada, se han convertido en una de las principales barreras en el sostenimiento del desarrollo alcanzando por los países expuesto-susceptibles (Obradovich, Tingley, \& Rahwan, 2018).

A esta vulnerabilidad social espacializada, se suma el incremento de eventos hidrometeorológicos a raíz de la intensificación del cambio climático en el último tiempo, representando casi el $90 \%$ de los eventos mundiales (Intergovernmental Panel on Climate Change [IPCC], 2014). Solo para el año 2018, de acuerdo con datos del Centro de Investigación sobre la Epidemiología de los Desastres (CRED), el impacto de eventos meteorológicos extremos afectó a 57.3 millones de personas, liderado por las inundaciones con una afectación de 35.4 millones (UNISDR, 2019).

Ante estos eventos, son las poblaciones con mayor vulnerabilidad social y con escasas oportunidades de acceso al desarrollo las que presentan menores capacidades de respuesta y recuperación, así como también un mayor impacto psicosocial negativo (Sandoval \& Fava, 2016; Romero \& Romero, 2015). De allí que se torne necesario instalar medidas que permitan reducir la vulnerabilidad y fortalecer las capacidades de afrontamiento, esto con el fin de reducir el riesgo de desastre (Gaillard, Cadag, \& Rampengan, 2019). En el ámbito psicosocial, si bien se han producido investigaciones sobre los efectos negativos individuales, como el estrés consecuente (Guerra, Plaza, \& Vargas, 2018; García, Vega, Briones, \& Bulnes, 2018), guías clínicas ante la emergencia (Cortes \& Figueroa, 2018; Errázuriz, Fischer, Behn, Letelier, \& Monari, 2019) y de crecimiento postraumático pos- desastre (García, Reyes, \& Cova, 2014; Wlodarczyk, Basabe, Páez, Villagrán, \& Reyes, 2017), se ha tendido a soslayar el papel agentivo de las poblaciones vulneradas (Astudillo \& Sandoval, 2019; Villagrán, Reyes, Wlodarczyk, \& Páez, 2014), sus capacidades de afrontamiento, el rol de la participación local y el empoderamiento comunitario (Gaillard et al., 2019; Sandoval et al. 2018; Wisner, 2016). Bajo esto, las estrategias colectivas de afrontamiento cobran vital trascendencia, tanto por la capacidad de empoderar a la comunidad ante la reducción del riesgo, como por la búsqueda participativa de soluciones locales compartidas (Campos, 2015; Espinoza, Espinoza, \& Fuentes, 2015).

En línea con lo anterior, el área de investigación que ha puesto especial atención en las capacidades de afrontamiento ha sido la de la resiliencia comunitaria (Atallah, Bacigalupe, \& Repetto, 2019; González-Muzzio, 2013), definida como la capacidad de autoorganización, aprendizaje y adaptación frente a riesgos socionaturales, en base a respuestas coordinadas y colectivas que en algunas ocasiones prescinden de las acciones institucionales. También ha sido estudiada como capital social, es decir, como aquellas acciones organizadas por la comunidad, en base a normas y redes, que logran movilizar recursos y respuestas frente a estos eventos (Loìpez-Cepeda, Tapia, \& Romero, 2017).

Dentro de estas líneas investigativas, la respuesta de la sociedad civil ante los afectados del desastre podría comprenderse como comportamiento prosocial entendido como «las conductas llevadas a cabo para ayudar o beneficiar a otros» (Sánchez-Queija, Oliva, \& Parra., 2006, p. 260), que puede o no ser altruista, abarcando una alta gama de actividades como comportamientos de rescate, asistencia, donación, apoyo social y voluntariado (Richaud, Mesurado, \& Cortada, 2012). Según Martí-Vilar, Corell-García y Merino-Soto (2019) estas acciones deben comprenderse independiente del tipo de motivación que guíe su actuar o del efecto que se produzca en otra persona en contraste al altruismo, el cual «supone cierto coste para quien las realiza y lo hace 
voluntariamente, con intención de lograr beneficios para otros y sin anticipar ninguna recompensa» (Guijo, 2002, p. 31).

Lombardini (2008) reconoce que la empatía es la base de este comportamiento, la cual se expresa en acciones de comprensión, refuerzo y soporte emocional (Auné, Blum, Abal, Lozzia, \& Attorresi, 2014). Por tanto, ante situaciones de desastre se generaría una empatía específica situacional, es decir, una experiencia situada afectiva-vicaria entre afectados y no afectados, fungiendo como motor de potenciales conductas humanitarias (Fuentes et al., 1993).

Para Caprara, Steca, Zelli y Capanna (2005) el origen de la conducta prosocial se asocia a un complejo desarrollo psicosocial, implicando procesos de atención, evaluación, razonamiento moral, competencia social y autorregulación de capacidades, requiriendo de cierto piso sociocognitivo. De acuerdo con Mora y Rojas (2010), estas conductas emergen desde una edad temprana, continuando su desarrollo/ fortalecimiento en distintos medios de socialización primaria o secundaria (Auné et al., 2014).

Ante situaciones de desastre, el comportamiento prosocial ha sido descrito como una respuesta de ayuda hacia la población afectada de tipo individual o colectiva (Clarke, 2002; Gantt, 2008). Estas iniciativas de ayuda son desplegadas transversalmente, principalmente por instituciones gubernamentales $\mathrm{y}$ grupos organizados de la sociedad civil, tales como organizaciones no gubernamentales o universidades (Kudó et al. 2005; Pérez, Lillo, Barrales, \& Astudillo, 2016). No obstante, parte de las primeras respuestas de ayuda es proporcionada por personas o grupos no organizados vinculados a las comunidades afectadas, esto mediante conductas espontáneas de facilitación de apoyo o de voluntariado (Moreno \& Shaw, 2018). Estos agentes actuarían de acuerdo a sus propias interpretaciones de las necesidades/satisfactores emergentes tras el desastre, lo cual puede transformarse en un obstáculo para las instituciones respondedoras o en acciones claves para la recuperación social (Auf der Heide, 2004). Por tanto, dado el rol activo de lo (inter)subjetivo de los actores involucrados ante el ciclo de riesgo-desastre, la presente investigación busca relevar la dimensión interpretativa de quienes buscan ayudar espontáneamente ante estas situaciones por medio del prisma de las teorías subjetivas (en adelante TS). Este enfoque define las TS como creencias particulares, supuestos o hipótesis que crean y mantienen las personas sobre sí mismas y su contexto con el objetivo de comprender y comunicarse, determinando la forma de planificación y acción posterior (Catalán, 2016; Flick, 2007). Además, según Cuadra-Martínez y Castro-Carrasco (2018), las TS son un término más inclusivo que permite estudiar las teorías personales explícitas o declaradas, como también las implícitas, obteniendo una visión más completa del fenómeno a conocer. Flick (2007) señala que las funciones de las TS son: a) definir una situación rápidamente, dando certeza en nuestra orientación de la realidad; b) entregar una explicación a lo sucedido; c) predecir eventos futuros; d) facilitar el desarrollo del comportamiento, permitiendo decidir entre diferentes alternativas conductuales; y e) estabilizar u optimizar la autovaloración.

Algunos estudios cualitativos previos sobre la dimensión subjetiva del comportamiento prosocial han dado cuenta de distintos factores facilitadores, tales como (i) la importancia del contexto valórico comunitario y las prácticas socioeducativas que este promueve (Cuadra et al., 2019), (ii) el papel de la asociatividad territorial y su función en la acción colectiva (Jerónimo \& Souza, 2015), (iii) el rol activo de la agencia en la movilización de capacidades de afrontamiento y de capital social de los lugares afectados (Sandoval, 2017), (iv) los significados/ sentidos diferenciados sobre la ayuda entre grupos (Magaña, Nadales, \& Rovira, 2010) y (v) la relevancia de la concienciación ambiental por medio de la educación (Cuadra-Martínez, Véliz-Vergara, SandovalDíaz, \& Castro, 2017). 
Respecto a tipologías de voluntariado, Marta, Pozzi y Marzana (2010) desde una perspectiva multidimensional identificaron cuatro tipos: (i) voluntarios en ejercicio, (ii) voluntarios por necesidades personales, (iii) exvoluntarios ciudadanos activos y (iv) exvoluntarios por oportunidad. Cadena (2010), por su parte, analiza dicha tipología desde las significaciones personales de estos, relevando el papel de las motivaciones en el involucramiento y permanencia en organizaciones prosociales, así como también, el intento de los jóvenes voluntarios por diferenciar causas y consecuencias de esta forma de participación. Respecto a las motivaciones se ha relevado (i) las características de las organizaciones donde participan, (ii) los efectos en la experiencia de ser voluntarios y (iii) otros aspectos personales y altruistas, tales como la autoestima, desarrollo propio, valores morales, reconocimiento social y empatía (Cuevas et al., 2015; Moreno \& Shaw, 2018).

A la luz de los antecedentes mencionados, la presente investigación tiene como objetivo principal conocer las teorías subjetivas de la conducta prosocial de jóvenes voluntarios durante las etapas de respuesta y rehabilitación de un desastre socionatural en Chile, el cual se desagrega en los siguientes objetivos específicos: a) analizar los sentidos subjetivos asignados a las acciones de ayuda; b) describir las explicaciones sobre el desarrollo, mantención e impacto de estas conductas e c) identificar los factores motivacionales de este tipo de comportamiento.

\section{Método}

\section{Tipo de estudio, metodología y diseño}

Corresponde a un estudio descriptivo-interpretativo de corte cualitativo, dado que se busca describir el comportamiento prosocial desde la propia subjetividad de los participantes (Flick, 2007). La investigación desarrolla un estudio de caso cualitativo entendido como el examen profundo y contextualizado de diversos aspectos de un mismo fenómeno (Coller, 2005). Para el presente caso se seleccionó un evento hidrometeorológico extremo ocurrido el 25 de marzo de 2015 (25M), el cual causó lluvias torrenciales y consecuentes aluviones de barro sobre la región de Atacama, registrando un total de 22 personas fallecidas, 28000 damnificados, 2000 viviendas destruidas y 5000 con daño mayor.

\section{Participantes}

Los participantes fueron 11 jóvenes de la comuna de Copiapó, de una población total de 30 registrados como voluntarios universitarios ante el desastre hidrometeorológico. Cabe destacar que este grupo de voluntarios se conformó al interior de la universidad para prestar ayuda durante el evento hidrometeorológico, no recibiendo ningún tipo de instrucción acerca de lo que constituye el comportamiento prosocial, sino más bien, acerca de cómo organizar el apoyo para la comunidad. La muestra quedó constituida por cinco estudiantes mujeres y seis hombres; siete de ellos poseen una edad de 18 a 24 años y tres de 25 a 30 años, pertenecientes a las carreras profesionales de Psicología y Kinesiología. Las entrevistas y grupos de discusión se aplicaron posemergencia (cinco meses posteriores al evento hidrometeorológico) y los participantes prestaron ayuda durante las etapas de respuesta y rehabilitación (aproximadamente 4 meses). Se utilizó un muestreo intencionado de tipo opinático (Ruiz, 2003) en base a la condición de voluntarios de los participantes. Como criterio de inclusión se consideró que (a) se encontraran matriculados en la universidad el año 2015 y (b) haber prestado ayuda a lo menos una semana. Como criterio de exclusión, se determinó descartar a aquellos estudiantes que no se encontraban registrados como voluntarios, esto para asegurar su condición de «voluntarios», la cual según Ríos (2004) implica: (a) no recibir recompensa por la ayuda prestada, (b) participar libremente en la actividad de ayuda, (c) beneficiar a otros con la ayuda y (d) accionar como voluntario en el marco de una organización. 


\section{Procedimiento de recolección de información}

Se contactó a los jefes de carrera respectivos, a quienes se les explicó el estudio, solicitándoles, a su vez, el registro de voluntarios. Posteriormente, se contactó a los estudiantes y se les explicó los fines del estudio, la metodología y los criterios éticos. Respecto de estos últimos, se consideró la (a) confidencialidad de la información entregada, (b) adecuado manejo de esta y el (c) carácter voluntario de su participación, solicitándoles firma de un consentimiento previo. Finalmente, se procedió a aplicar los instrumentos de producción de información en instalaciones de la universidad. En primer lugar, se realizaron las entrevistas semiestructuradas, dado que estas permiten acceder de manera detallada y profunda en la subjetividad del tema abordado, para posteriormente aplicar el grupo de discusión, mediante el cual fue posible contrastar la información obtenida y acceder a TS colectivas (Catalán, 2016).

\section{Instrumentos}

En primera instancia se aplicaron entrevistas semiestructuradas, puesto que permiten utilizar preguntas abiertas como guías para que al momento de retomar una idea, se pueda profundizar en la temática central de forma paulatina (Flick, 2007). Su duración fue en promedio de una hora, realizando un total de seis entrevistas. Se cauteló la representatividad de casos, correspondiendo a estudiantes de ambos sexos, cuatro de la carrera de Kinesiología (3H; $1 \mathrm{M})^{1}$ y dos a la carrera de Psicología (1H; 1M). El guion temático consideró como ejes indagatorios: a) sentidos subjetivos asignados a las acciones de ayuda; b) explicaciones sobre el desarrollo, mantención e impacto de estas conductas y c) factores motivacionales de este tipo de comportamiento.

En una segunda instancia se llevó a cabo un grupo de discusión, al cual se dio inicio entregando un «estímulo de debate», para posteriormente introducir preguntas aclaratorias y de profundización (Flick,

$\mathrm{H}=$ Hombre $; \mathrm{M}=$ Mujer
2007). Duró en promedio una hora y se realizó en las dependencias de la universidad a la que asisten los estudiantes. Contó con cuatro participantes: tres de la carrera de Psicología $(2 \mathrm{H} ; 1 \mathrm{M})$ y uno de Kinesiología (1M), rigiéndose por el mismo guion temático de la entrevista. Respecto del número de participantes del grupo de discusión, para Pérez y Víquez (2010) debe oscilar entre cinco y diez, a fin de evitar que se transforme en un monólogo o en una conversación íntima. En este caso, se cauteló que los participantes no se conocieran entre ellos a fin de disminuir estas limitaciones (Arboleda, 2008).

\section{Procedimiento de análisis de datos}

El análisis de datos se inició con las transcripciones de las entrevistas, lo que permitió reconocer temáticas a profundizar para contrastar, posteriormente, mediante el grupo de discusión (Catalán, 2016). Se utilizó el proceso de codificación de la teoría fundamentada, utilizando la versión straussiana «orientada a un trabajo de investigación más enraizado en la descripción interpretativa que en la construcción de teoría formal emergente» (Carrero, Soriano, \& Trinidad, 2012, p. 19). De esta forma, se realizaron dos tipos de codificaciones: la (a) codificación abierta, cuyo proceso analítico es identificar conceptos, datos, propiedades y dimensiones, elaborando códigos representativos de las oraciones, párrafos y frases del texto transcrito. Las TS se identificaron conjuntamente entre los investigadores, mediante los enunciados del tipo si entonces, porque, esto conduce a aquello (Catalán, 2016; Flick, 2007). El paso siguiente fue (b) la codificación axial, que consiste en «depurar y diferenciar las categorías derivadas de la codificación abierta»(Flick, 2007, p. 197), seleccionando las que parecieron más representativas del problema de estudio y estableciendo relaciones entre las categorías axiales y sus subcategorías. Se obtuvieron tres de estas: (a) el sentido subjetivo de la conducta prosocial ante el desastre; (b) desarrollo, mantención e impacto de la conducta prosocial y (c) motivaciones de la conducta prosocial. 


\section{Resultados}

El sentido subjetivo de la conducta prosocial en situación de desastre socionatural

La empatía es una dimensión importante del comportamiento prosocial ante desastres, siendo significada como la capacidad de ponerse en el lugar del otro respecto al impacto del evento, incentivando la movilización de ayuda en quienes fueron percibidos como afectados en comparación con ellos mismos.

... hay que preocuparse un poco más del otro y ver; ponerse en el zapato del otro (E2) ${ }^{2}$.

Yo creo que es más preocupación por el otro, aun viendo que mi casa está con barro, pero voy a ayudar al que está con barro completamente. Era día 25, día de pago, y muchos de mis vecinos no tenían mercadería porque no le habían pagado, recuerdo que entre todos hacíamos ollas comunes en la villa y así nos ayudábamos (E3).

Los participantes, si bien conciben la conducta prosocial como un proceso ante situaciones de desastre, su despliegue conlleva cierta caducidad temporal reducida a la etapa de emergencia; por lo que en una interpretación de segundo orden, esta conducta podría quedar en un estado de latencia ante el retorno a la «cotidianidad».

Puedes ayudar un rato, unos meses y después te olvidas, y no pasó nada... por lo menos se debe preguntar a la persona cómo está, cómo se siente; son cosas mínimas, pero valen en el momento en el que ha pasado tanta tragedia, de lo contrario sería como que no pasó nada y no hay nada que preguntar (E4).

... son lazos con fecha de caducidad porque después que pasó el aluvión creamos un grupo de WhatsApp y ahora todos pelean por puras cosas pequeñas: porque no cierran el portón, porque no hacen esto... son lazos que nacieron en el momento de la catástrofe y después desaparecieron (E2).
El comportamiento de ayuda también es considerado como gratuito y pareciera ser ofensivo el intentar remunerarlo, puesto que se busca entregar un apoyo desinteresado hacia quienes son percibidos como vulnerados por el desastre. No obstante, consideran positiva la gratificación mediante la entrega de recursos que apunten a satisfacer necesidades simbólicas o valóricas (e.g., autoestima), existiendo cierto beneficio colateral por ayudar.

... me llamó mucho la atención cuando me dijeron «oye, pero no tengo plata», a lo que le respondí: «no caballero no se preocupe, si no estoy cobrando, no le voy a cobrar tampoco» (E1).

... es como la acción de "pucha, quizás yo necesito esta botella de agua más que tú, pero te paleteaste, toma» dando a entender que de verdad agradezco el gesto que tuviste y te lo entrego, acto desinteresado por parte de ellos, quizás... (GD, P1).

Complementariamente, si bien estas conductas no emergen de una planificación formal previa, su despliegue es enmarcado como parte de la organización colectiva emergente de ayuda, permitiendo realizar un trabajo colaborativo, de organización incipiente y de mayor cobertura hacia la población afectada.

... fue un trabajo organizado, es decir, uno sacaba barro, el otro lo apilaba en las cubetas, otro sacaba las cubetas, otro carreteaba y el otro iba haciendo un drenaje hacia fuera para que fuera saliendo el barro y el camión pasara y se lo llevara. Fue un trabajo bien, bien organizado y bacán, se avanzó rápido, se sacó súper rápido el barro, porque estaban todos organizados (E4).

Para los participantes, la conducta prosocial implica un refuerzo y soporte emocional, debido a que la ayuda material se ve acompañaba de una conversación respecto a lo ocurrido, facilitando procesos y sentidos

$2 \quad$ E = Entrevistado; GD = Grupo de discusión; $\mathrm{I}=$ Investigador; $\mathrm{P}$ = participante 
compartidos para una conexión empática entre voluntarios y afectados.

... un trabajo colectivo con las personas, sobre todo a nivel emocional. Se conectaron muchas personas con las demás y esto es importante, si no tienes conexión con los demás, ayudas muy aisladamente. Si no te afecta lo que pasa con los demás, entonces para qué ayudas (E4).

Finalmente, consideran que es necesario diferenciar la verdadera ayuda de aquella que parece satisfacer más bien intereses egocéntricos propios de un volunturismo ${ }^{3}$ iatrogénico o de donaciones inservibles al señalar que ante situaciones de crisis, existirían acciones que no buscan generar apoyo auténtico hacia los afectados.

... no y ahí me chocó el hecho de que ellas venían de Valparaíso y ayudaban, no sé, cinco minutos y se tomaban una foto, cinco minutos otra foto, cinco minutos otra foto y a mí me molestó eso... (E1).

...a mí me pasó que nos tocaba, por ejemplo, doblar ropa interior, y ropa interior sucia, entonces es así... «¡cómo cresta (carajo) manda ropa así!»... yo pensaba "iqué persona!, como tan poca humanidad»... (GD, P1).

\section{Desarrollo, mantención e impacto de la conducta prosocial en situación de desastre socionatural}

\section{Desarrollo}

Los participantes consideran que existe un (a) desarrollo biográfico de la conducta prosocial asociado a los valores que adquirieron en las instituciones de socialización, tales como la familia, la escuela o la iglesia, de la que formaron parte en algún momento.

... valores más que nada, de la casa, de la familia y todos los valores que empiezan desde chico. Si tú no adquieres valores desde chico ya es muy difícil que los aprendas cuando grande (E6).

Para que esta conducta pueda activarse ante el desastre, explican que es necesario (b) valorar el grado de riesgo en que se encuentran otros significativos (familiar o amigo). Esto implica reconocer el condicionamiento de la conducta de ayuda hacia otros «desconocidos» tipificados como vulnerados, solo cuando otros significativos no se encuentran afectados por el desastre.

... en mi caso fue así, yo no podía estar pensando en otra cosa si no sabía que mi papá estaba vivo, no podía entregar la ayuda de manera adecuada si tenía mi mente en otra parte. Para mí, mi entorno tiene que estar sano y de ahí salir (GD, P1).

Por último, otro factor de desarrollo de esta conducta fue la (c) percepción insuficiente de ayuda gubernamental ante el desastre, generando un sentimiento de insatisfacción institucional y de responsabilidad personal, ante lo cual decidieron actuar.

... La ayuda fue mucho más privada que de las mismas instituciones políticas, por ejemplo, pasaban las camionetas [municipio] que son esas azules y pasaban grabando... Claro, porque si no me ayuda, qué voy a hacer, tengo que hacerlo yo, que tengo que esperar a la autoridad si la autoridad ni siquiera me puede ayudar (E2).

\section{Mantención}

Los participantes consideran como un factor mediador de la conducta prosocial (a) la colaboración conjunta junto a un otro significativo, puesto que esto posibilita un soporte emocional basado en el «optimismo», así como también, en el refuerzo de la «seguridad personal» al momento de entregar ayuda a otros.

El volunturismo serían todas aquellas colaboraciones a corto plazo que se realizan aprovechando un viaje cuyo principal objetivo era el turismo (Mendes \& Sonaglio, 2013). 
... Sí, estar con amigos ayuda, sirve harto para que uno siga y no pierda el optimismo (GD, P2).

... por un tema de seguridad, yo al menos fui con un grupo de amigas y todo, pero igual era un tema importante para mi protección, porque uno no sabía qué podría pasarle en cualquier lado (GD, P1).

También explican como un mantenedor de la prosocialidad la (b) empatía situacional, ya que existía la necesidad inmediata en dejar habitables las viviendas afectadas por la inundación. En este caso, si bien existe una experiencia compartida del desastre, esta es vivenciada de forma diferenciada entre quienes fueron dañados directamente y quiénes no.

... En realidad, creo que fue como algo mutuo entre mi polola (novia) y yo, como que los dos nos tirábamos para arriba. Como veíamos que el asunto había sido malo, que había gente que la estaba pasando mal, había que ayudar no más (E1).

\section{Impacto}

Respecto al impacto del comportamiento prosocial tras el evento, para los participantes esta conducta posibilitó un afrontamiento positivo activo, facilitando el inicio de la etapa de reconstrucción material, esto ante el lento actuar de las autoridades e instituciones gubernamentales respondedoras.

... la Universidad de Magallanes, por ejemplo, en Twitter ponía: «Tengo, aproximadamente dos camiones gigantes de ayuda y no sé cómo hacerlos llegar», esto porque la municipalidad tampoco hacía mucha conexión. Así que nosotros los trajimos y fuimos a entregar con ellos a Paipote, a Los Loros, igual que con la INJUV de La Serena. Nunca pensamos que se iba a generar tantas redes para ayudar a la gente, cuando ya empiezas a ver que funciona este tema del acopio y de la ayuda, ya uno no para hasta que más o menos se estabiliza la situación (E5).
Por otro lado, los participantes consideran que las conductas prosociales desplegadas presentaron (b) un impacto positivo en su identidad personal, facilitando sentimientos de bienestar subjetivo y gratitud al reconocer la importancia de la ayuda de otros.

... es como rara la sensación, porque en el momento tú no piensas en cómo activas la sensación de ir y ayudar. Pero lo sentí bien porque cada vez que iba a ayudar decía: «la humanidad no está tan perdida», por así decirlo, y seguía ayudando. Decía: «oye, si te falta algo, yo te ayudo», es como altruismo por así llamarle, pero con más sentimientos... (E5).

... yo encuentro que fue lo mejor que pude haber hecho en esta situación, porque de alguna forma, como te mencionaba, se produjo como una misma sanación del trauma que podía haber producido la situación, aparte que te entrega como una satisfacción personal poder ayudar a otros (E2).

Finalmente, los participantes señalaron que, debido a las amplias consecuencias del desastre sobre las distintas dimensiones de la vida cotidiana, el evento produjo (c) una especie de libertad relativa situacional para poder ir en ayuda de otros, en el sentido de que su propia rutina diaria se encontraba interrumpida.

... no teníamos nada que hacer, yo no tenía redes sociales, no tenía luz, no tenía nada, entonces estaba el tiempo, si no teníamos nada que hacer, estaba la disponibilidad de ir en ayuda... (GD, P3).

\section{Motivaciones de la conducta prosocial en situación de desastre socionatural}

\section{Motivación extrínseca}

Entre los factores externos que llevaron al actuar prosocial, se encontraba la (1) percepción de la magnitud de la emergencia, en términos de extensión y daños causados, esto a partir de lo observado y de los relatos recibidos por parte de las personas afectadas. 
... ya ahí nos dimos cuenta de que sí se había salido el río, y me imaginé todo para allá y dije: «ya estamos». Entonces tratamos de comunicarnos entre todos y fuimos a ayudar y ahí cachamos (entendimos) lo que había pasado (E3).

Los participantes explican que (2) vivenciar la necesidad de las personas vulneradas, provocó en ellos un tipo de afectación colateral negativa, generando la necesidad de otorgar ayuda para así reducir su ansiedad.

Claro, cómo olvidar la situación, ver que hay gente que está más necesitada, que tiene más problemas, que necesita más de tu ayuda que uno mismo (E4).

Claro, en parte lo que tú comentas y también el ver que todas las personas están pasando por lo mismo, o sea, si no te afectó a ti de manera colateral igual te afectan las personas que están a tu alrededor; entonces, de cierta forma, técnicamente, estás siendo afectado. Aquí sufrió toda la población, no solo un sector, en ese sentido sí creo que movió eso (E5).

\section{Motivación intrínseca}

Un motivador intrínseco de la conducta prosocial es (b) la satisfacción personal que les generaba el poder entregar asistencia al otro, a pesar de que esto tenía ciertos costes personales como el cansancio y el agotamiento.

... era larga la jornada, porque era desde las ocho de la mañana, después llegaban las cinco para bañarme, dormir y después, al otro día, lo mismo. Al principio dije: «No, no voy a poder», pero al otro día me levantaba muy bien, porque sabía que estaba ayudando. Terminé la jornada, pero fue genial la sensación, fue bonita (E1).

... sentías el cariño de las personas que estaban alrededor cuando estabas ayudando, como que te inyectaba más energía (E4).
Por último, los participantes explican que el hecho de no ser afectados directamente por el desastre es un factor motivador para ellos, ya que (c) su estado de bienestar personal les permitía ir en ayuda de los otros.

... yo estoy bien, tengo mis piernas, mis manos buenas, entonces puedo ir a ayudar, obviamente me voy a ir (E1).

... en parte eso, esa fue mi motivación a ayudar: estaba tan cómodo, estábamos bien, que tenía que hacer algo (GD, P1).

\section{Discusión}

La Figura 1 esquematiza la teoría subjetiva del comportamiento prosocial ante el desastre de jóvenes voluntarios universitarios de Atacama, Chile. Según esta, lo prosocial se expresa bajo un repertorio de conductas espontaneas de ayuda humanitaria, acotadas a las etapas de respuesta y rehabilitación; la cual es asumida como una responsabilidad éticapersonal, tanto por la empatía hacia quienes han sido vulnerados por el desastre, como por la percepción de incapacidad ante la respuesta institucionalizada (Cuevas et al., 2015; Sandoval, 2017). A su vez, con el despliegue de estas acciones de sentido humanitarioético, se acompaña una sensación de reducción de emociones negativas personales (pena y angustia por los afectados) y un incremento de sensaciones positivas (reconocimiento y gratitud). Por tanto, los jóvenes presentan un comportamiento de voluntariado de tipo híbrido, actuando como (i) agentes activos de la ciudadanía, pero también (ii) movidos por necesidades personales (Cadena, 2010; Marta et al., 2010); esto bajo el telón de fondo de un fuerte sentido de pertenencia hacia el territorio común afectado (Jerónimo \& Souza, 2015).

Utilizando la taxonomía de González (2000), podríamos clasificar la presente conducta prosocial del tipo de (a) ayuda directa, (b) no solicitada por el benefactor, (c) de benefactor identificable, (d) de 
temporalidad reducida y (e) no planificada. Complementario a esto, se identificaron dos dimensiones principales, del tipo (a) afectivacognitiva: vinculada a la capacidad de empatizar (Lombardini, 2008), reducción de ansiedad personal (Batson \& Powell, 2003), gratitud, responsabilidad y bienestar (Caprara et al., 2005) y valoración relativa del riesgo; así como la dimensión (b) agentiva situacional acotada a la emergencia, vinculada a la valoración de la gravedad del impacto del desastre para el potencial despliegue de acciones (González, 2000), la percepción de (in)capacidades de respuesta institucional (Sandoval \& Fava, 2016) y de fortalecimiento de la identidad social (Batson \& Powell, 2003; Cadena, 2010; Sandoval, 2017). Esto refuerza la idea que el comportamiento prosocial funciona en base a un árbol de decisiones, relevando la importancia de la planificación de esta (Marín, 2009; Moreno \& Shaw, 2018).

En cuanto a los condicionantes de estas acciones de ayuda, identificamos el rol de los juicios evaluativos ante el riesgo de ayudar y la importancia del soporte de figuras significativas (Cuevas et al., 2015). Respecto a esto último, cuando los voluntarios espontáneos corroboran que sus grupos significativos se encuentran bien, lo cual se ve acompañado de una evaluación de ayuda institucional insuficiente ante el desastre, existe una mayor posibilidad de implicación de un comportamiento prosocial; sin embargo, esta implicación conlleva una evaluación socioafectiva de los riesgos ante el involucramiento de la situación, considerando sus capacidades, su expectativa de autoeficacia y sentido de responsabilidad (Caprara et al., 2005; Marín, 2009).

En segundo lugar, el desarrollo del comportamiento prosocial estaría vinculado a una formación valórica temprana, aprehendida biográficamente durante la infancia y la adolescencia, a través de la familia o instituciones como la escuela e iglesia (Auné et al., 2014; Mora \& Rojas, 2010). Esto releva la importancia del papel formativo de los contextos socioeducativos, formales e informales, de las prácticas sociales y de sentido que allí se promueven (Cuadra et al., 2017; Cuadra et al., 2019).

En términos de su mantención, de acuerdo a los resultados, este dependería tanto por la persistencia de estas actividades en grupos afines, de la empatía situacional hacia los afectados y de un proceso reflexivo continuo (Cadena, 2010; Marta et al., 2010). Al respecto, es importante considerar que el voluntariado se entiende como una estrategia de participación social que redunda en un beneficio para la comunidad. Por tanto, esta capacidad individual, a su vez, podría servir de base para el fortalecimiento local de la resiliencia comunitaria (Atallah et al., 2019; González-Muzzio, 2013) y de capital social (Loìpez-Cepeda et al., 2017) en busca de la necesidad de diseñar planes de acción colectivos a partir de los conocimientos, recursos y valores presentes en los territorios expuesto-susceptibles (Gaillard et al., 2019; Sandoval et al., 2018). En esta misma línea, se ha encontrado que el comportamiento prosocial fomenta la creación de vínculos sociales positivos entre las personas que habitan un mismo lugar, fortaleciendo aún más el sentido de comunidad luego de un desastre (Astudillo \& Sandoval, 2019; González-Muzzio, 2013; Villagrán et al., 2014), así como también el crecimiento postraumático posterior (Wlodarczyk et al., 2017).

En tercer lugar, los participantes identifican motivaciones extrínsecas e intrínsecas para ayudar, vinculando las primeras a las condiciones de vulnerabilidad social emergentes tras el desastre; mientras que las intrínsecas se asocian al bienestar personal, tanto de corte hedonista como eudemonista (Cuevas et al., 2015; Moreno \& Shaw, 2018; Sandoval, 2014). Cadena (2010) reconoce estas motivaciones eudemonistas como positivas, contribuyendo al desarrollo de la identidad social y conllevando, a quienes ejercen estas acciones, el desarrollo de una ciudadanía activa.

En cuarto lugar, a nivel práctico se desprenden las siguientes orientaciones: (a) la importancia de 
formalizar el rol de los voluntarios ante situaciones de desastre (Moreno \& Shaw, 2018), esto con el objetivo de reducir el entorpecimiento de estos actores ante las labores institucionales de emergencias (Auf der Heide, 2004) como del lamentable turismo del desastre (Mendes \& Sonaglio, 2013); (b) la importancia de considerar la educación como un factor clave en el desarrollo y aprendizaje de la prosocialidad (Campos, 2015), no solo en el contexto escolar formal, sino que también a nivel comunitario (Cuadra et al., 2017; Cuadra et al., 2019; Sandoval et al., 2018); (c) la necesidad de que este proceso educativo considere el aprendizaje formativo sobre gestión de emergencias, etapa en la cual tienden a actuar los voluntarios, esto con el objetivo de instalar capacidades disposicionales reflexivas acerca de cómo implementar una ayuda integral y efectiva ante situaciones de crisis (Cortes \& Figueroa, 2018; Errázuriz et al., 2019; Kudó et al., 2005).
Por último, respecto a la opción teóricametodológica, los participantes describen la conducta prosocial ante desastres en base a sus TS, lo que no solo contribuye a comprender la problemática, sino también al programa de investigación de las mismas (Catalán, 2016; Cuadra-Martínez \& Castro-Carrasco, 2018; Kindermann \& Riegel, 2016). A su vez, se recomienda que en posteriores investigaciones pudiesen estudiar el comportamiento prosocial en voluntarios institucionalizados con el objetivo de profundizar en la participación y mantenimiento de esta conducta en el tiempo (Arias, 2015; Cadena, 2010; Marta et al., 2010). Asimismo, seguir profundizando en la conducta prosocial en jóvenes, ya que esta genera satisfacción personal, favorece vínculos y podría reforzar la autoestima, suponiéndose así un soporte vital para la prevención de trastornos psíquicos ante este tipo de crisis (Guerra et al., 2018; Martínez, Inglés, Piqueras, \& Oblitas, 2010).

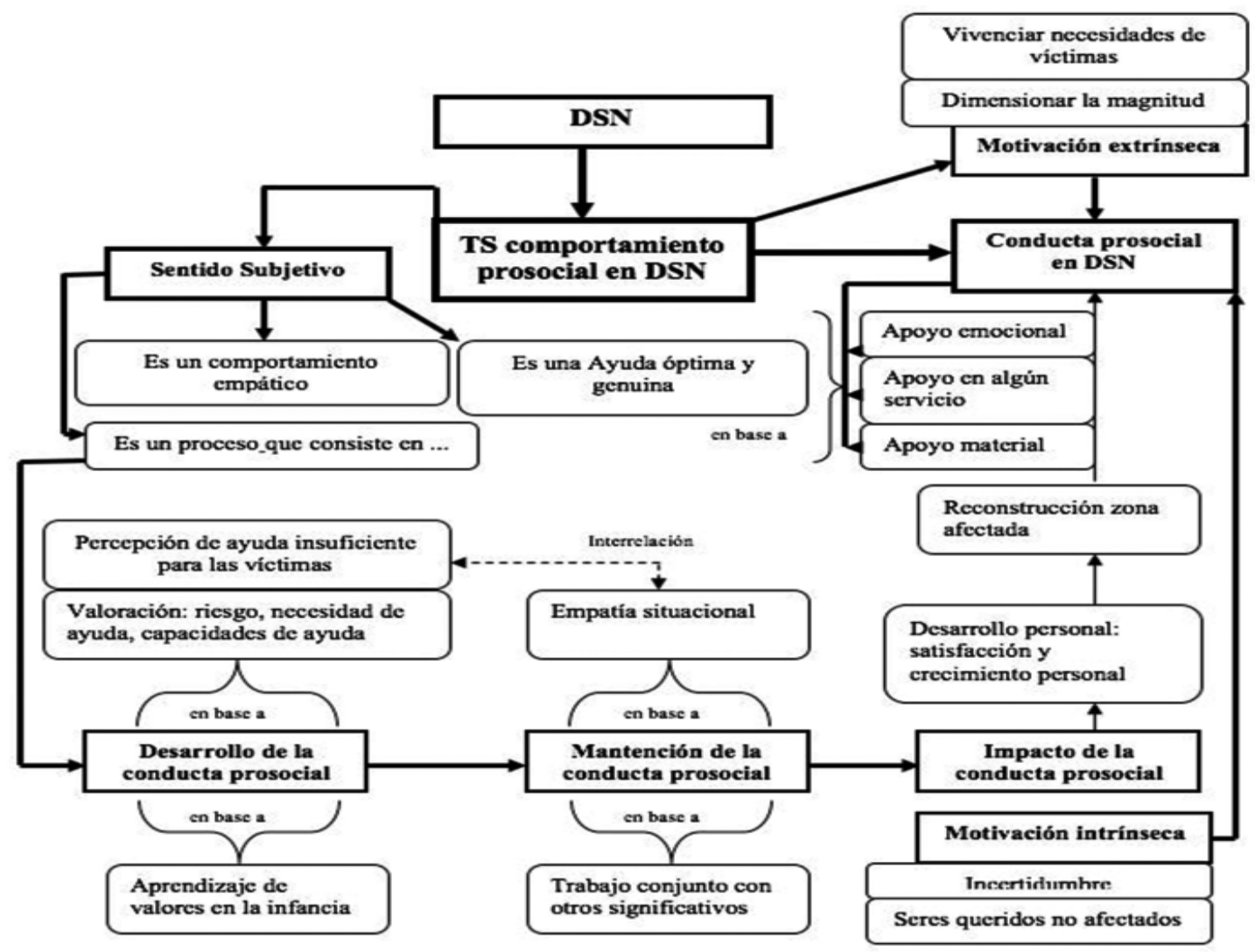

Figura 1. Teoría subjetiva del comportamiento prosocial ante desastres socionaturales DSN = Desastre socionatural; TS = Teorías subjetivas. 


\section{Conflicto de intereses}

Los autores declaran que no hay ningún conflicto de intereses.

\section{Responsabilidad ética}

Previa producción de los datos, los participantes debieron firmar un consentimiento informado como herramienta de responsabilidad ética evitando en todo momento la visualización y difusión de los datos de los participantes durante el estudio y en la elaboración del artículo.

\section{Contribución de autoría}

RA: recolección de datos, análisis cualitativo y escritura inicial.

CP: recolección de datos, análisis cualitativo y escritura inicial.

NY: recolección de datos, análisis cualitativo y escritura inicial.

DC: concepción y diseño del estudio, interpretación de los datos y revisión final del manuscrito.

JS: discusión teórica, análisis cualitativo y revisión final del manuscrito.

\section{Referencias}

Arboleda, L. (2008). El grupo de discusión como aproximación metodológica en investigaciones cualitativas. Rev. Fac. Nac. Salud Pública, 26(1), 6977. Recuperado de https://www.redalyc.org/articu lo.oa?id=12026111

Arias, W. (2015). Conducta prosocial y psicología positiva. Avances en Psicología: Revista de la Facultad de Psicología y Humanidades, 23(1), 3747. doi: 10.33539/avpsicol.2015.v23n1.169

Astudillo. F, \& Sandoval, J. (2019). Justicia espacial, desastres socionaturales y políticas del espacio: dinámicas sociopolíticas frente a los aluviones y proceso de recuperación en Copiapó, Chile. Revista
Colombiana de Geografía, 28(2), 303-321. doi: 10.1 5446/rcdg.v28n2.73520

Atallah, D., Bacigalupe, G., \& Repetto, P. (2019). Centering at the Margins: Critical Community Resilience Praxis. Journal of Humanistic Psychology. 1-31 doi: 10.1177/0022167818825305

Auf der Heide, E. (2004). Common Misconceptions about Disasters: Panic, the Disaster Syndrome and Looting. In M. O'Leary (Ed.), The first 72 hours: A Community Approach to Disaster Preparedness (pp. 337-377). Lincoln, EE. UU.: iUniverse.

Auné, S., Blum, D., Abal, F., Lozzia, G., \& Attorresi, F. (2014). La conducta prosocial: estado actual de investigación. Perspectivas en Psicología, 11(2), 2133. Recuperado de http://www.seadpsi.com.ar/revist as/index.php/pep/article/view/153

Batson, C, \& Powell, A. (2003). Altruism and Prosocial Behavior. Handbook of Psychology, 463-484. doi: 10.1002/0471264385.wei0519

Cadena, M. (2010). Aportes y desafíos de estudiar el voluntariado desde la mirada del sujeto: Análisis de los hallazgos de Marta, Pozzi y Marzana (2010). Psykhe, 19(2), 19-23. doi: 10.4067/S0718-22282 010000200003

Campos, A. (2015). El desafío de la prevención psicosocial de desastres: construcción y práctica para el desarrollo de un enfoque integral. In C. Arteaga \& R. Tapia (Eds.), Vulnerabilidades y desastres socionaturales: Experiencias recientes en Chile, (pp. 169-180). Santiago, Chile: Editorial Universitaria.

Caprara, G., Steca, P., Zelli, A., \& Capanna, C. (2005). A New Scale for Measuring Adults' Prosocialness. European Journal of Psychological Assessment, 21(2), 77-89. doi: 10.1027/1015-5759.21.2.77

Carrero, V., Soriano, R, \& Trinidad, A. (2012). El desarrollo de teoría desde la generalización conceptual. Madrid: Centro de Investigaciones Sociológicas.

Catalán, J. (2016). Hacia la formulación de una teoría general de las teorías subjetivas. Psicoperpectivas. Individuo y Sociedad, 15(1), 53-65. doi: 10.5027/p sicoperspectivas-Vol15-Issue1-fulltext-739 
Clarke, L. (2002). Panic: Myth or reality? Contexts, 1(3), 21-26. doi: 10.1525/ctx.2002.1.3.21

Coller, X. (2005). Estudios de casos. Madrid: Centro de Investigaciones Sociológicas.

Cortes, P., \& Figueroa, R. (2018). Manual ABCDE para la aplicación de Primeros Auxilios Psicológicos: En crisis individuales y colectivas. Chile, PUC/ CIGIDEN. Recuperado de https://medicina.uc.cl/ publicacion/manual-abcde-la-aplicacion-primerosauxilios-psicologicos/

Cuadra-Martínez, D., Sandoval-Díaz, J., Pérez-Zapata, D., Castro-Carrasco, P., Véliz-Vergara, D., GuzmánÁvalos, J., \& Ramos-Thompson, G. (2019). Helping One's Neighbor: Teaching and Learning Prosocial Behavior in a Religious Community. Religions, 10(9), 515, 1-17. doi: 10.3390/rel10090515

Cuadra-Martínez, D., \& Castro-Carrasco, P. (2018). Cambio educativo: propuesta de un modelo de transformación de teorías subjetivas. Liberabit, 24(1), 147-164. doi: 10.24265/liberabit.2018.v24n1.10

Cuadra-Martínez, D., Véliz-Vergara, D., Sandoval-Díaz, J., \& Castro-Carrasco, P. (2017). Aportes a la economía ecológica: una revisión de estudios latinoamericanos sobre subjetividades medio ambientales. Psicoperspectivas. Individuo y Sociedad, 16(2), 156-169. doi: 10.5027/psicoperspectivas-vol16-iss ue2-fulltext970

Cuevas, M., Marrero, M., Moreno, I., Santini, E., Valentin, J., \& Ocasio, A. (2015). Motivación de un grupo de voluntarios afiliados a dos organizaciones sin fines de lucro en el área sur de Puerto Rico. Informes Psicológicos, 15(1), 47-61. doi: 10.18566/infpsic v15n1a03

Errázuriz, P., Fischer, C., Behn, A., Letelier, C., \& Monari, M. (2019). Orientaciones prácticas para psicoterapeutas que atienden a pacientes con TEPT después de un desastre natural. Psykhe, 28(1), 1-13. doi: 10.7764/psykhe.28.1.1218

Espinoza, A., Espinoza, C., \& Fuentes, A. (2015). Retornando a Chaitén: diagnóstico participativo de una comunidad educativa desplazada por un desastre socionatural. Magallania, 43(3), 65-76. doi: 10.4 067/S0718-22442015000300006
Flick, U. (2007). Introducción a la investigación cualitativa. Madrid, España: Ediciones Morata.

Fuentes, M., López, F., Etxebarria, I., Ledesma, A., Oortiz, M., \& Apocada, P. (1993). Empatía, Role-taking y concepto de ser humano, como factores asociados a la conducta prosocial/altruista. Infancia $y$ Aprendizaje, 16(61), 73-87. doi: 10.1080/02103 702.1993.10822365

Gaillard, J., Cadag, J., \& Rampengan, M. (2019). People’s capacities in facing hazards and disasters: an overview. Natural Hazards, 95(3), 863-876. doi: 10.1007/s11069-018-3519-1

García, V. (2005). El riesgo como construcción social y la construcción social de riesgos. Desacatos, 19, 1124. doi: $10.29340 / 19.1042$

García, F., Reyes, A., \& Cova, F. (2014). Severidad del trauma, optimismo, crecimiento postraumático y bienestar en sobrevivientes de un desastre natural. Universitas Psychologica, 13(2), 575-584. doi: 10.1 1144/Javeriana.UPSY13-2.stop

García, F., Vega, N., Briones, F., \& Bulnes, Y. (2018). Rumiación, crecimiento y sintomatología postraumática en personas que han vivido experiencias altamente estresantes. Avances en Psicología Latinoamericana, 36(3), 443-457. doi: 10.12804/rev istas.urosario.edu.co/apl/a.4983

Gantt, P. (2008). Hazardous materials: Regulations, response and site operations ( $2^{\mathrm{da}}$ ed.), Florence, KY: Delmar Cengage Learning.

González-Muzzio, C. (2013). El rol del lugar y el capital social en la resiliencia comunitaria posdesastre. Aproximaciones mediante un estudio de caso después del terremoto del 27/F. EURE, 39(117), 25-48. doi: $10.4067 /$ S0250-71612013000200002

González, M. (2000). Conducta prosocial: evaluación e intervención. Madrid, España: Ediciones Morata.

Guerra, C., Plaza, H., \& Vargas, J. (2018). Estrés postraumático en adolescentes expuestos a un mega incendio: Asociaciones con factores cognitivos y emocionales. Psicoperspectivas. Individuo y Sociedad, 17(2), 175-186. doi: 10.5027/psicopers pectivas-vol17-issue2-fulltext-1213 
Guijo, V. (2002). Estudio multifactorial de la conducta prosocial en niños de cinco y seis año (Tesis doctoral). Universidad de Burgos, España. Recuperado de https://core.ac.uk/download/pdf/61545294.pdf

Intergovernmental Panel on Climate Change. (2014). Climate change 2014: Impacts, Adaptation, and Vulnerability. IPCC's Fifth Anual Report. Ginebra, Suiza: Intergovernmental Panel on Climate Change.

Jerónimo, R., \& Souza, R. (2015). Environmental Psychology: A Study on the Resistance to Mining in Içara, SC. Psicologia \& Sociedade, 27(1), 80-86. doi: 10.1590/1807-03102015v27n1p080

Kindermann, K., \& Riegel, U. (2016). Subjective Theories of Teachers: Variations and Methodical Modifications of a Research Program. Forum: Qualitative Social Research, 17(2), 1-34. doi: 10.1 7169/fqs-17.2.2486

Kudó, I., Velasquéz, T., Iza, M., Ángeles, A., Pezo, C., \& Martínez, P. (2005). Una experiencia de intervención en crisis en el sur del Perú: Brigadas Psicológicas. Revista de Psicología, 23(2), 295-336. Recuperado de http://revistas.pucp.edu.pe/index.php/psicologia/ article/view/2154

Loìpez-Cepeda, J., Tapia, A., \& Romero, H. (2017). Capital social y respuestas a perturbaciones ambientales en la comunidad andina de Caquena, norte de Chile. Interciencia, 42(7), 430-436. Recuperado de https://www.interciencia.net/wpcontent/uploads/2017/08/430-5991-LOPEZ-Cepeda42-7.pdf

Lombardini, J. (2008). Empatía paterna y nuevo modelo de paternidad. Psicodebate: Psicología, Cultura y Sociedad, 9, 81-92. Recuperado de https://dialnet.u nirioja.es/servlet/articulo?codigo $=5645282$

Magaña, I., Nadales, S., \& Rovira, R. (2010). Catástrofe, subjetividad femenina y reconstrucción: aportes y desafíos desde un enfoque de género para la intervención psicosocial en comunidades afectadas por el terremoto. Terapia Psicológica, 28(2), 169177. doi: 10.4067/S0718-48082010000200005

Marín, J. (2009). Conductas prosociales en el barrio Los Pinos de la ciudad de Barranquilla, Colombia. Revista CES Psicología, 2(2), 60-75. Recuperado de http:// revistas.ces.edu.co/index.php/psicologia/article/view/ $897 / 588$

Marta, E., Pozzi, M, \& Marzana, D. (2010). Volunteers and Ex-Volunteers: Paths to Civic Engagement Through Volunteerism. Psykhe, 19(2), 5-17. doi: 10.4 067/S0718-22282010000200002

Martí-Vilar, M., Corell-García, L., \& Merino-Soto, C. (2019). Revisión sistemática de medidas de conducta prosocial. Revista de Psicología, 37(1), 349-377. doi: 10.18800/psico.201901.012

Martínez, A., Inglés, C., Piqueras, J., \& Oblitas, L. (2010). Papel de la conducta prosocial y las relaciones sociales en el bienestar psíquico y físico del adolecente. Avances en Psicología Latinoamericana, 28(1), 74-84. Recuperado de https://dialnet.unirioja.e s/servlet/articulo?codigo $=3329331$

Mendes, T., \& Sonaglio, K. (2013). Volunturismo: uma abordagem conceitual. Turismo: Visão e Ação, 15(2), 185-206. Recuperado de http://www.redalyc.org/ articulo.oa?id=261056071003

Mora, G., \& Rojas, M. (2010). Estudio de la conducta prosocial en niños(as) de preescolar y programa educativo para su desarrollo (Tesis de grado). Universidad de Los Andes, Venezuela.

Moreno, J., \& Shaw, D. (2018). Guía de orientaciones para la gestión de voluntarios espontáneos en situaciones de emergencia y desastre. Provincia de Concepción, región del Bío Bío, Chile. Recuperado de https://adm.talcahuano.cl/archivos/gestion_riesgo/ descargas/eCoMa0W7vxNiNVs4K3wE_Plan_VEs_ Concepcio\%cc\%81n_LR.pdf

Oficina de las Naciones Unidas para Reducción de Riesgo de Desastres. (2015). Marco de Sendai para la Reducción del Riesgo de Desastres 2015-2030. Ginebra, Suiza: UNISDR.

Oficina de las Naciones Unidas para Reducción de Riesgo de Desastres. (23 de enero de 2019). 2018: 60 millones de personas resultaron afectadas por diversos eventos meteorológicos extremos. Recuperado de https://eird.org/americas/noticias/201 8-60-millones-de-personas-resultaron-afectadas-pordiversos-eventos-meteorologicos-extremos.html\#. XatY65NKg_V 
Obradovich, N., Tingley, D., \& Rahwan, I. (2018). Effects of environmental stressors on daily governance. Proceedings of the National Academy of Sciences of the United States of America, 115(35), 8710-8715. doi: 10.1073/pnas.1803765115

Pérez, S., Lillo, M. P., Barrales, S. B., \& Astudillo, V. (2016). Organizaciones de la Sociedad Civil en contexto de desastre: entre la subjetividad y la norma. Revista Sul-Americana de Psicología, 4(2), 343368. Recuperado de http://ediciones.ucsh.cl/ojs/ index.php/RSAP/article/view/1789

Pérez, R., \& Víquez, D. (2010). Los grupos de discusión como metodología adecuada para estudiar las cogniciones sociales. Actualidad en Psicología, 23(110), 87-101. doi: 10.15517/ap.v23i110.12

Richaud, M., Mesurado, B., \& Cortada, A. (2012). Analysis of Dimensions of Prosocial Behavior in an Argentinean Sample of Children. Psychological Reports, 111(3), 687-696. doi: 10.2466/10.11.17.P R0.111.6.687-696

Ríos, R. (2004). Universitarios y voluntariado: análisis del involucramiento en acciones filantrópicas de los alumnos de la PUC. Psykhe, 13(2), 99-115. doi: 10.4 067/S0718-22282004000200008

Romero, H., \& Romero, H. (2015). Ecología política de los desastres: vulnerabilidad, exclusión socioterritorial y erupciones volcánicas en la patagonia chilena. Magallania, 43(3), 7-26. doi: 10.4067/S071 8-22442015000300002

Ruiz, J. (2003). Metodología de la investigación cualitativa. Bilbao: Universidad de Deusto.

Sánchez-Queija, I., Oliva, A., \& Parra, A. (2006). Empatía y conducta prosocial durante la adolescencia. Revista de Psicología Social, 21(3), 259-271. doi: 10.1174/ 021347406778538230
Sandoval, J. (2014). La subjetividad en el enfoque del desarrollo: calidad de vida, bienestar subjetivo y capacidades. Revista Interdisciplinaria de Filosofía y Psicología, 9(30), 35-48. Recuperado de http:// limite.uta.cl/index.php/limite/article/view/133

Sandoval, J. (2017). Dimensión subjetiva de la vulnerabilidad ante riesgos y desastres socionaturales: cuatro casos de estudio (Tesis de doctorado). Universidad de Chile, Santiago, Chile.

Sandoval, J., \& Fava, D. (2016). Significados y vulnerabilidad social ante el terremoto y tsunami del 27 de febrero del 2010: la dimensión subjetiva desde los damnificados. Summa Psicológica, 13(2) 23-32. Recuperado de https://summapsicologica.cl/index.p hp/summa/article/view/306

Sandoval, J., Rojas, L., Villalobos, M., Sandoval, C., Moraga, F., \& Aguirre, N. (2018). De organización vecinal hacia la gestión local del riesgo: diagnóstico de vulnerabilidad y capacidad. Revista INVI, 33(92), 155-180. doi: 10.4067/S0718-83582018000 100155

Villagrán, L., Reyes, C., Wlodarczyk, A., \& Páez, D. (2014). Afrontamiento comunal, crecimiento postraumático colectivo y bienestar social en el contexto del terremoto del 27 de febrero de 2010 en Chile. Terapia Psicológica, 32(3), 243-254. doi: 10.4 067/S0718-48082014000300007

Wisner, B. (2016). Vulnerability as Concept, Model, Metric, and Tool. Oxford Research Encyclopedia of Natural Hazard Science, 1-51. doi: 10.1093/acrefo re/9780199389407.013.25

Wlodarczyk, A., Basabe, N., Páez, D., Villagrán, L., \& Reyes, C. (2017). Individual and Collective Posttraumatic Growth in Victims of Natural Disasters: A Multidimensional Perspective. Journal of Loss and Trauma, 22(5), 371-384. doi: 10.1080/153250 24.2017.1297657 
Robert Alvarado Ardiles

Universidad Santo Tomás, Chile, sede Copiapó

Psicólogo. Línea de investigación: comportamiento prosocial en situaciones críticas

ORCID: 0000-0002-7688-2041

shawn-6-crahan@hotmail.com

Constanza Pradenas Ossandón

Universidad Santo Tomás, Chile, sede Copiapó

Psicóloga. Línea de investigación: comportamiento prosocial en situaciones críticas

ORCID: 0000-0002-6156-0293

constanzap.ossandon@gmail.com

Nátaly Yañez Vega

Universidad Santo Tomás, Chile, sede Copiapó

Psicóloga. Línea de investigación: comportamiento prosocial en situaciones críticas

ORCID: 0000-0003-1571-632X

nyanezsalcedo@yahoo.es

David Cuadra Martínez

Universidad de Atacama, Chile.

Psicólogo, magíster en Psicología con mención educacional. Académico de la Universidad de Atacama, Facultad de Humanidades y Educación, Departamento de Psicología. Línea de investigación: subjetividad y educación, formación profesional y comportamiento prosocial. ORCID: 0000-0002-0810-2795

david.cuadra@uda.cl

José Sandoval Díaz

Universidad del Bío Bío, sede Chillan.

Doctor en Psicología, Investigador del Centro Estudios de Ñuble y Grupo de Investigación Género, Ciudadanía y Equidad (GI 170424/ EF), Universidad del Bío-Bío. Línea de investigación: capacidades de afrontamiento ante riesgos y desastres socionaturales, vulnerabilidad educativa.

ORCID: 0000-0001-7247-7113.

Autor corresponsal: jsandoval@ubiobio.cl 